\title{
Semibatch operation and primary cyclization effects in homogeneous free-radical crosslinking copolymerizations
}

\author{
Rolando C.S. Dias ${ }^{\mathrm{a}, 1}$, Mário Rui P.F.N. Costa ${ }^{\mathrm{b}, *}$ \\ ${ }^{a}$ LSRE-Instituto Politécnico de Bragança, Quinta de S. Apolónia, 5300 Bragança, Portugal \\ ${ }^{\mathrm{b}}$ Departamento de Engenharia Química, LSRE-Faculdade de Engenharia da Universidade do Porto, Rua Roberto Frias s/n, 4200-465 Porto Codex, Portugal
}

\begin{abstract}
A theoretical analysis is presented on the use of semibatch reactors for producing non-linear copolymers through the homogeneous freeradical polymerization of mono- and divinyl-monomers. The kinetic scheme distinguishes two kinds of pendant double bonds and five kinds of polymer radicals, as it also takes into account the primary cyclization of terminal divinyl units. In spite of the fairly large number of groups and reactions, the generating function of vector chain length distribution of polymer species can still be accurately computed by a numerical implementation of the method of characteristics and thus average molecular weights before and after gelation can be reliably obtained. The influence of feed policies of the monomers, initiator and transfer agent on gelation, average chain lengths and weight fraction of sol is discussed, as well as their sensitivity to some kinetic parameters.
\end{abstract}

Keywords: Crosslinking; Semibatch; Cyclization

\section{Introduction}

\subsection{Aims of present work}

This work is a sequel of previous publications by the same authors on the modeling of non-linear free-radical polymerization, checking its applicability in two new situations:

- semibatch reactor modeling;

- non-negligible cyclization reactions.

In spite of the large number of reactions needed to describe these chemical systems (even if only a sub-class of cyclization reactions is taken into account in a rigorous way), a fairly appealing mathematical treatment will be

\footnotetext{
* Corresponding author. Tel./fax: +351225081666.

E-mail addresses: rdias@ipb.pt (R.C.S. Dias), mrcosta@fe.up.pt (M.R. P.F.N. Costa).

${ }^{1}$ Fax: +351 273313051 .
}

shown to lead to the prediction of sol fraction and its average molecular weights with current, low-end computer hardware and public domain software.

There are many issues concerning the modeling of chemical reactions in these systems, which are briefly discussed below. The approach here presented is intended only to make easier the description of reactions and thus estimation of kinetic parameters, to carry out the extrapolation of batch reactor behavior to semibatch operation.

\subsection{Modeling of non-linear free-radical polymerization: a summary of the state-of-art}

In the last two decades, the synthesis of crosslinked networks with controlled structure has become a subject of increasing interest. This is driven by their extensive use in medicine, agriculture and sanitary industries, due to unique properties of the resulting polymer gels such as their ability to absorb many times their dry weight of solvent.

Several experimental works can be found in the literature about batch copolymerization of mono- and divinylmonomers of different chemical compositions and polymerization conditions [1-6]. Other recent works in this 
subject $[7,8]$ are examples witnessing the experimental efforts on studying the formation of crosslinked networks.

Simultaneously with these experimental efforts, numerous computational studies have also been carried out in order to quantitatively describe free radical crosslinking copolymerizations: the well-known probabilistic approach [9], the method of the moments [10-16], Monte Carlo simulation [17-25] or statistical crosslinking methods [26] are some examples of techniques used in this context. 'Numerical fractionation' [27,28] and real chain-length domain calculation exploiting Galerkin finite elements method $[29,30]$ are alternative techniques for dealing with non-linear free radical polymerizations.

Based upon a general kinetic model for dealing with nonlinear irreversible copolymerizations [31] the authors have recently made some improvements in modeling non-linear free radical polymerizations with different chemical systems and reactors [32-35]. The main advantage of this method is its ability to deal with non-linear free radical copolymerizations with the elimination of a set of approximation conditions with widespread use in the modeling of this kind of systems. Moreover, the method can be applied past gel point and is based on a generic notation allowing the analysis of different systems in an automated way. In these works, an extensive comparison of the predictions of present method with the pseudo-kinetic method, Monte Carlo method, different versions of the method of the moments and with numerical fractionation, was carried out.

In this work, the analysis of batch free radical crosslinking copolymerizations is extended to semibatch reactors. A general situation where the reagents fed to reactor are the two monomers, initiator, the solvent and possibly a chain transfer agent is considered; other operation conditions are a sub-set of this one. The influence of different feed policies in the gelation and properties of the resulting networks is analyzed. As far as the authors know, this is the first attempt to simulate semibatch reactors past gel point. The possible description of cyclization reactions is an additional advantage of the proposed method, but only a simplified model has been considered in this paper.

\section{Chemical groups and kinetic scheme}

The kinetic scheme considers the usual reactions in freeradical polymerizations (initiator decomposition, addition of primary radicals from initiator or chain transfer agents to double bonds, propagation of monomers and pendant double bonds with the different kinds of macroradicals, terminations by combination and disproportionation and transfers to solvent and a chain transfer agent). Although important in allyl polymerizations [36], transfers to monomer (as well as transfer to initiator) have been neglected; they could nevertheless be easily added.

Propagation reactions in this system also includes primary cyclization by intramolecular propagation of a terminal free-radical with the contiguous double bond, forming an $\alpha$-cycle. They are schematically presented in Fig. 1. Notice this is a somewhat rare situation. We will nevertheless go through it in detail and try to re-use it for more general situations.

According to our approach, equations are written for a generic kinetic scheme, and the first task in its application is the definition of the chemical groups and chemical reactions intervening in the polymerization process [31,34]. There is a total number of 20 chemical groups $A_{j}, 12$ of them belonging to the polymer: five kinds of free-radical sites (Fig. 1) and two kinds of pendant double bonds (with and without contiguous free-radical site). The remaining five groups in polymer are 'dead' polymerized fragments, one of which is the $\alpha$-cycle. The detailed description of the groups and reactions is not presented here by conciseness but is available free of charge from the authors as supplementary material.

Even neglecting intramolecular propagations leading to larger cycles (and at the origin of micro-gel) already an appreciable number of elementary reactions (76) result. To take into account intramolecular propagation or mutual termination of free-radicals occurring between reacting groups belonging to different repeating units (named secondary cyclization when there are cross-links in the chain connecting the intervening groups and primary otherwise), the number of groups and reactions would increase by some tens after collecting all the new reactions in such a scheme. It is not known whether the model equations can still be solved with the same computer hardware, although it is likely they would still do if only moments before gel point are sought.

If most cyclization reactions are primary and there is not a great change of reactivity of pendant double bonds in the small loops formed, then this model might be used, but the cyclization rate constant becomes an apparent value depending on the fraction of divinyl monomer.

It is known that values of initiator efficiency, chain termination rate coefficients and even propagation rate coefficients can vary significantly with polymer concentration because of diffusional hindrance. Chain length dependence of apparent termination rate constant of free radicals is known to exist and to follow a complex pattern $[37,38]$. These variations can be incorporated in the set of differential equations by expressing rate coefficients as functions of monomer conversion(s) and average molecular weight of polymer, yielding good predictions with copolymerizations with little or no branching [39]. The phenomenon becomes much harder to model when divinyl monomers are present, as branching very effectively hinders bulk diffusion of polymer molecules, thus making necessary a thorough revision of the above cited approach as far as termination reaction is concerned. Inadequacy of classical kinetics without including the effect of average chain length on termination before establishment of reaction diffusion 


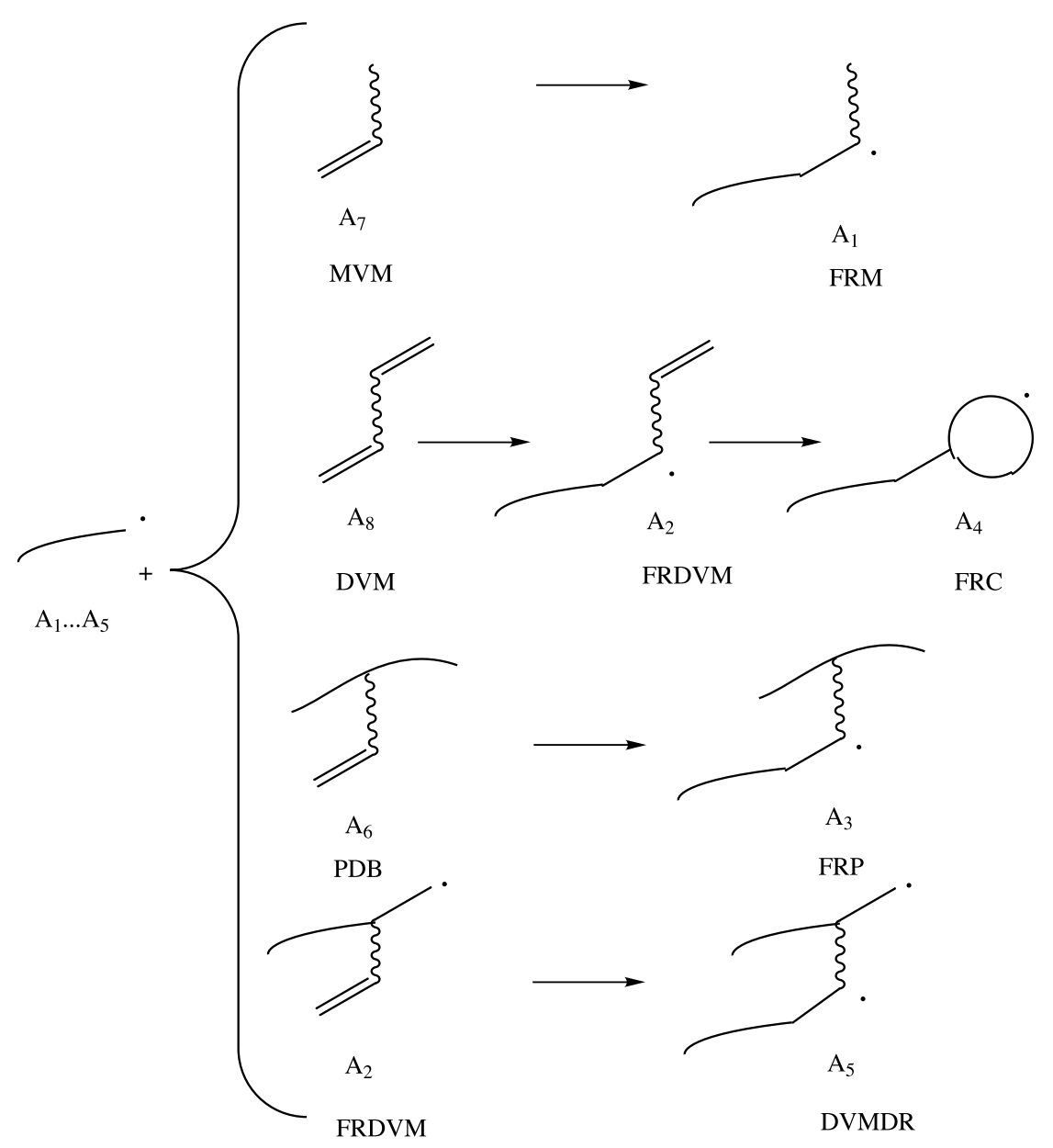

Fig. 1. Propagation reactions in vinyl/divinyl monomer free-radical polymerization considered in this work.

regime has been recently proved [40]. Several research groups (Ref. [41] and works cited therein) have also observed that a relatively large concentration of trapped free-radicals remains stable in the absence of oxygen for several days without being able to propagate or terminate.

Thermodynamic effects ('excluded volume effect') often lead to the decrease of the reactivity of pendant double bonds and free-radicals $[42,43]$. The polymerization media should be described as multiphasic; otherwise, rate coefficients will become once again apparent values, depending on medium composition.

The change of rate coefficients during the polymerization can be inserted in the differential set of equations of the present model by expressing these rate coefficients as functions of conversion or excess Van der Waals volume fraction or other kind of correlation. Nevertheless, in this work, we only intend to show the ability of this approach to predict average molecular weights and sol fraction with non linear free radical polymerizations in semibatch reactors, assuming that batch reaction can be successfully modelled. For this purpose, isothermal reactors and constant rate coefficients are assumed.

\section{Mathematical modeling of semibatch reactors}

The main principles of the mathematical model used in this work have already been presented $[31,34]$ as well as the particular analysis of free-radical crosslinking copolymerization in batch reactor [32]. In this case, the relevant equations of the model were explicitly written. A similar chemical system had been considered, but chain transfer agent CTA and solvent ' $\mathrm{S}$ ' are now introduced in this case study. The influence of these new components can be easily incorporated by following the generic model, and therefore, we will focus the presentation in the particularities related with semibatch operation.

The mass balance equation for the moment generating function of the chain length distribution (CLD) is a first order partial differential equation, which for a semibatch reactor can be written as Eq. (1):

$\frac{\partial G}{\partial t}=G_{R_{\mathrm{P}}}+\frac{Q_{\mathrm{F}}(t)}{V(t)}\left[G_{\mathrm{F}}(t)-G\right]-R_{\mathrm{v}} G$

where $G_{R_{\mathrm{P}}}$ is the generating function of the rate equation of formation of polymer species by chemical reaction, which is obviously the right-hand side of the mass balance equation 
for the moment generating function in a batch reactor. In this equation, $Q_{\mathrm{F}}(t)$ represents the inlet flow-rate and $V(t)$ the reaction volume, which can be obtained from Eq. (2):

$\frac{\mathrm{d} V}{\mathrm{~d} t}=R_{\mathrm{v}} V+Q_{\mathrm{F}}(t)$

where $R_{\mathrm{v}}$ is the volume change ratio due to density changes, evaporation or precipitation of by-products. In this work, these effects will be considered negligible and then $R_{\mathrm{v}}=0$ in what follows.

$G_{R_{\mathrm{P}}}$ is a function involving the concentration of the reactive chemical groups (in the present case $A_{j}$ with $j=$ $1, \ldots, 14)$, a vector with the Laplace parameters of the generating function for the active groups that are present in the polymer $\left(s_{j}\right.$ with $\left.j=1, \ldots, 6\right)$ and a correspondent vector of the partial derivatives of $G$ with respect to $\log s_{j}: G_{j}^{\prime}=$ $\partial G / \partial \log s_{j}$ with $j=1, \ldots, 6$. In a compact form they can be written as $G_{R_{\mathrm{P}}}=G_{R_{\mathrm{P}}}\left(\mathbf{A}, \mathbf{s}, \mathbf{G}^{\prime}\right)$ where $\mathbf{A}$ is a vector of dimension 14 and $\mathbf{s}, \mathbf{G}^{\prime}$ are vectors of dimension 6 .

The solution of Eq. (1) is obtained by the method of characteristics, leading to a system of ordinary differential equations which must be solved to obtain the moment generating function of CLD is compactly represented by Eqs. (3)-(6):

$\frac{\mathrm{d} A_{j}}{\mathrm{~d} t}=R_{A_{j}}(\mathbf{A})+\frac{Q_{\mathrm{F}}}{V}\left(A_{j_{\mathrm{F}}}-A_{j}\right) \quad j=1, \ldots, 14$

$\frac{\mathrm{d} G_{j}^{\prime}}{\mathrm{d} t}=R_{G_{j}^{\prime}}\left(\mathbf{A}, \mathbf{s}, \mathbf{G}^{\prime}\right)+\frac{Q_{\mathrm{F}}}{V}\left(G_{j_{\mathrm{F}}}^{\prime}-G_{j}^{\prime}\right) \quad j=1, \ldots, 6$

$\frac{\mathrm{d} s_{j}}{\mathrm{~d} t}=s_{j}^{\prime}\left(\mathbf{A}, \mathbf{s}, \mathbf{G}^{\prime}\right) \quad j=1, \ldots, 6$

$\frac{\mathrm{d} G}{\mathrm{~d} t}=R_{G}\left(\mathbf{A}, \mathbf{s}, \mathbf{G}^{\prime}\right)+\frac{Q_{\mathrm{F}}}{V}\left(G_{\mathrm{F}}-G\right)$

In above equations, $R_{A_{j}}(\mathbf{A}), R_{G_{j}^{\prime}}\left(\mathbf{A}, \mathbf{s}, \mathbf{G}^{\prime}\right), s_{j}^{\prime}\left(\mathbf{A}, \mathbf{s}, \mathbf{G}^{\prime}\right)$ and $R_{G}\left(\mathbf{A}, \mathbf{s}, \mathbf{G}^{\prime}\right)$ are the expressions for the same polymerization system in batch reactor. It should be noticed that in spite of Eq. (5) not being involved with the inlet terms, the solution for the vector $\mathbf{s}$ is not the same as for batch reactor due to the influence of $\mathbf{A}$ and $\mathbf{G}^{\prime}$ in $s_{j}^{\prime}\left(\mathbf{A}, \mathbf{s}, \mathbf{G}^{\prime}\right)$.

At the start of polymerization, the reactor can be charged with prescribed amounts of the two monomers (monovinyl monomer MVM and divinyl monomer DVM) with concentrations $M_{1_{0}}$ and $M_{2_{0}}$, initiator I, CTA and solvent S, and so the initial conditions for Eq. (3) are given by Eqs. (7)-(12):

$A_{7_{l t=0}}=M_{1_{0}}$

$A_{8_{l=0}}=M_{2_{0}}$

$A_{9_{\mid t=0}}=I_{0}$

$A_{10_{\mid t=0}}=\mathrm{CTA}_{0}$
$A_{11_{l=0}}=S_{0}$

$A_{j_{t=0}}=0 \quad j=1 \ldots 6,12,13,14$

In the beginning of the reaction, there is no polymer in the reactor and so the initial conditions for Eqs. (4) and (6) become Eqs. (13) and (14):

$G_{j_{l=0}}^{\prime}=0 \quad j=1 \ldots 6$

$G_{\mid t=0}=0$

The initial value $\mathbf{s}_{0}$ of the vector of dummy Laplace variables $\mathbf{s}$ has to be computed as a function of its final value, $\mathbf{s}_{\mathrm{f}}$, which is prescribed for a certain time of polymerization $t=t_{\mathrm{f}}$. The initial conditions for Eq. (5) can therefore be obtained from Eq. (15):

$s_{j_{l=0}}=s_{j_{0}} \quad j=1 \ldots 6$

The specification of the reactor feed composition is done in a similar way. The feed contains the same reagents initially charged to the reactor, and in the same way as for the initial conditions, nearly all variables assume a nil value in the feed with the possible exceptions of those given by Eqs. (16)(20) below:

$A_{8_{\mathrm{F}}}=M_{1_{\mathrm{F}}}$

$A_{9_{\mathrm{F}}}=M_{2_{\mathrm{F}}}$

$A_{10_{\mathrm{F}}}=I_{\mathrm{F}}$

$A_{11_{\mathrm{F}}}=\mathrm{CTA}_{\mathrm{F}}$

$A_{12_{\mathrm{F}}}=S_{\mathrm{F}}$

The detailed resolution of the problem of the characteristics and its numerical implementation have been discussed in our previous works [32-34]. It has also been advised therein to avoid writing explicitly the differential equations for the moments of the CLD, as it is a time-consuming and errorprone task. It is preferable to use the developed generic computer program, which performs this procedure automatically. The same option will be used in the present work.

From now on, we focus the presentation in discussing the influence of the initial charge of the reactor (Eqs. (7)-(11)) and feed policies (Eqs. (16)-(20)) on the dynamics of the average properties of the polymer network. Different simulations will be also presented by varying the inlet flow rate, $Q_{\mathrm{F}}(t)$ and the influence of the kinetic scheme will be also analyzed by considering the primary cyclization reaction.

\section{Kinetic parameters used in simulations}

It is not our purpose to discuss in depth the complex problem of kinetic modeling and parameter estimation for 
these chemical systems, but a few hints will be given in order to assess the applicability of this approach. For a real chemical system, rate data and molecular weights should be first collected for the homopolymerizations, leading to independent estimation of propagation and termination rate parameters. Prediction of initiation efficiency changes seems to be fairly well described thanks to incorporating recent knowledge on polymer/solvent diffusion [39]. However, a more fundamental question is whether the description of termination reactions by a chemical kinetic rate equation is indeed adequate.

For generality, no particular chemical system has been selected for this study, but the chosen kinetic parameters should allow a simulation as far as possible close to the real behavior of crosslinking copolymerization systems. A basic set of kinetic parameters will be fixed in the calculations in order to have a realistic time evolution of the copolymerization; they are collected in the Supplementary material.

The reference rate coefficients for monomer initiation, propagation and termination are the same as in the homopolymerization of styrene at $60{ }^{\circ} \mathrm{C}$ [1]. It is known that for styrene the main mechanism of termination is by combination and that for acrylates and methacrylates both combination and disproportionation should occur. In this work, it is considered that termination is $50 \%$ for each mechanism. The reference chain transfer agent constant $\left(C_{\mathrm{CTA}}\right)$ corresponds to the system styrene/carbon tetrabromide and for chain transfer to solvent $\left(C_{S}\right)$ the value for the system styrene/benzene was chosen. The kinetic constant of initiator decomposition ( $f$ and $k_{\mathrm{d}}$ ) is valid for AIBN, but no change of $f$ with conversion will be considered.

With the exception of kinetic parameters for homopolymerizations and reactivity ratios for copolymerizations, most of the information needed for the considered model is not available. There is not (yet) enough information from quantitative structure-activity studies to predict the remaining ones.

In the propagation reactions are involved five kind of macroradicals and four different monomers: two real monomers MVM and DVM plus two kinds of pendant double bonds, those with a contiguous free-radical site FRDVM and those without (PDB). If the cyclization reaction is neglected, the system can be simplified as the copolymerization of three monomers (two real monomers plus lumped pendant double bonds) with three kinds of macroradicals. In this situation, a parallelism with terpolymerization can be identified.

So, besides initiator efficiency, 75 rate coefficients have to be known, owing to the great variety of free-radicals sites and double bonds considered in this work. Polymer composition data in batch reactors have to be quite detailed. Values of propagation rate constants would have to be estimated from C13 NMR measured sequence lengths, using C13-enriched divinyl monomer in order to overcome low signal strength, no positive evidence of loop formation having been found with current conditions [44].

ESR spectra can sometimes be used to measure the concentrations of free-radicals, but this technique is only successful with certain chemical systems.

The relative values of rate constants of transfer to solvent or CTA should also be measurable by analyzing the endgroups by C13-NMR.

Little is known about cross-termination rate constants (30 of them, as there are five kinds of free-radicals, terminating either by combination or dismutation): only PLP studies combined also with C13 NMR to measure the dyads formed by termination can shed some light about these parameters. Notice, however, that knowledge of propagation and transfer is more critical to the kinetic modeling of these systems, since in principle chain transfer events should be more frequent than termination when a CTA is used.

The six rate constants of the addition of primary radicals (from I, S or CTA) to the two monomers or pendant double bonds should not have a great influence on the rate of monomer consumption or molecular weights unless they are much less than propagation rate constants, which is not usual. Accordingly, it should be acceptable to consider them equal to the propagation rate constants of monovinyl monomer terminated free-radicals.

With the exception of the radical reactivity ratio of the monovinyl monomer, which can be easily experimentally determined from polymer composition data, the other reactivity ratio is much more difficult to obtain through that technique due to the small amount of divinyl monomer in the initial reaction mixture. The value of $r_{12}=k_{\mathrm{p} 11} / k_{\mathrm{p} 12}=$ 0.44 for the system styrene/divinylbenzene will be used as Ref. [1].

Many rate coefficients will have to be fixed with the help of qualitative observations which are usually accepted for this kind of systems.

In the propagation of pendant double bonds with freeradical sites from monovinyl monomer FRM, a decrease of reactivity by a factor of 0.1 is supposed to occur relatively to the homopropagation of MVM with FRM. The reactivity of pendant double bonds with different kinds of radicals is supposed to be the same, regardless of the presence of a contiguous radical site. The propagation of MVM with the free-radical from divinyl monomer FRDVM is supposed to decrease by a factor of 10 and a relation $r_{12} r_{21}=0.1$ is assumed in the same way as a non-ideal copolymerization. A further decrease of the reactivity of PDB and the radical FRC formed by cyclization with FRDVM by a factor of 0.01 is also considered. An additional decrease by a factor of 0.01 is assumed for the reactions involving the free-radical from pendant double bonds FRP. The reactivity of the FRC radicals is supposed to be one half of the correspondent propagations with FRP. Propagations of the two radicals sites in the di-radical species DVMDR were assumed to have same rate constants as FRDVM. 
For the initiation reactions, as above stated, it is assumed that the rate parameters involved are the same as in the correspondent propagation of the different kinds of double bonds with FRM. For instance, the rate coefficient for the initiation of PDB by the primary free-radical PRC formed from chain transfer agent is assumed to be the same as for the propagation of PDB with FRM.

For the chain transfer reactions the ratio $C_{\mathrm{CTA}}=$ $k_{\mathrm{CTA} i} / k_{\mathrm{p} i 1}=2.2$ is assumed to be the same for all polymer free-radicals. This means that the chain transfer rate coefficient for each free-radical in polymer can be computed from the value of its rate of propagation with MVM. An analogous rule was used to set the rate coefficients of transfer to solvent: $C_{\mathrm{S}}=k_{\mathrm{si}} / k_{\mathrm{p} i 1}=2.3 \times 10^{-6}$.

In order to take into account the likely dependence of the rate of termination with the kind of polymer free-radicals involved, the following simple relation is considered: $k_{\mathrm{tc}_{i j}}=2 k_{\mathrm{tc}} /\left(i^{2}+j^{2}\right)$. This implies a decrease in the termination rate coefficient in the sequence FRM, FRDVM, FRP and FRC $(i=1,2,3,4)$. Once again, this is a matter to be solved by appropriate experimental studies. The rate coefficients of intermolecular terminations for DVMDR are considered to be the same as FRDVM. If the rate coefficients for the homoterminations of the different radicals were known, the cross-terminations could be obtained, as usually accepted, through the correspondent geometric mean or a similar relation. Nevertheless, lacking suitable information in this subject and for simulation purposes, the used termination constants seem plausible.

For the modeling of primary cyclization, two kinds of approaches can be found in the literature: cyclization is either considered to occur at a constant rate $[13,45-48]$ or it is treated as a bimolecular reaction (with kinetic constant usually equal to propagation) by distinguishing the local radicals from the bulk radicals $[22,49]$.

In the present work, a purely kinetic approach is used: cyclization is considered as an unimolecular process, and therefore reactions between macro-radicals and contiguous pendant double bonds occur with kinetically determined rates. Thus, it is not possible to directly use the kinetic information about cyclization available in the literature.

A batch copolymerization with $y_{M_{2}}=0.05$ (around 50\% in volume of solvent) was considered for the estimation of the unimolecular kinetic constant $k_{\text {cyc }}$ in order to observe a fraction of pendant vinyls consumed by primary cyclization (FCYC) in the experimental range determined by Landin and Macosko [45]. The value $k_{\mathrm{cyc}}=2 \mathrm{~s}^{-1}$ was obtained by considering that the FCYC should be around $0.04[13,45]$ at gel point for a batch operation with $y_{M_{2}}=M_{2_{0}} / M_{1_{0}}=0.05$. Notice that the value of FCYC is not constant along the polymerization.

This estimate of $k_{\mathrm{cyc}}$ is strongly affected by the remaining kinetic parameters, mainly the free-radical and pendant double bonds relative reactivities. In this context, with $k_{\mathrm{cyc}}=1,2$ and $5 \mathrm{~s}^{-1}$ the values $\mathrm{FCYC}=0.02,0.04$ and 0.1 , respectively, were obtained. Delays of the gel time (relatively to the absence of cyclization) of $0.55,1.15$ and $3.20 \mathrm{~h}$, respectively, are predicted in those situations. With the kinetic parameters and operation conditions (dilution) fixed in this work, in order to reproduce FCYC values around 0.3 a value of $k_{\text {cyc }}=20 \mathrm{~s}^{-1}$ should be used and several hours (around $19 \mathrm{~h}$ ) of delay in the gel time is therefore predicted. So, values of $k_{\mathrm{cyc}}$ in the range of $1-5 \mathrm{~s}^{-1}$ seem plausible.

Intramolecular termination of DVMDR is obviously possible. The magnitude of this rate constant is quite uncertain. As the concentration of DVMDR is likely to be very low, it should not visibly affect the course of polymerization.

\section{Batch operation}

The batch operation of this system will be used as a basis for the analysis of the semibatch operation. For this reason, some simulations concerning free radical cross-linking copolymerizations in batch were also performed. Without loss of generality, the physical properties of the reagents considered in this work are those correspondent to the system styrene/divinylbenzene/AIBN/carbon tetrabromide/benzene (see the Supplementary material).

A better understanding of the influence of semibatch operation and primary cyclization in the polymer properties is obtained by an initial isolation of these two effects. Therefore, in the next sections are presented simulations for different feed policies of the reactants without primary cyclization. These results can be obtained by setting $k_{\mathrm{cyc}}=0$ in the kinetic scheme before presented or by considering a simplified kinetic scheme where three kind of macroradicals are present and two real monomers plus a single (lumped) kind of pendant double bonds are polymerized. The numerical results obtained with the two approaches are obviously the same and this fact was used to confirm the consistency of the model.

The time evolution of number- and weight-average molecular weights in a batch reactor were obtained for different initial compositions of the system. The concentration of monovinyl monomer and initiator have been kept fixed as $M_{1_{0}}=4 \mathrm{~mol} \mathrm{dm}^{-3}$ and $I_{0}=0.08 \mathrm{~mol} \mathrm{dm}^{-3}$ and the initial content in divinyl monomer is expressed by the parameter $y_{M_{2}}=M_{2_{0}} / M_{1_{0}}$. In these conditions, the solvent concentration is around $S_{0}=5.6 \mathrm{~mol} \mathrm{dm}^{-3}$. The influence of the CTA in gelation of the system can be simulated in a similar way, and as expected, gelation is delayed by increasing the content of CTA which is represented by $y_{\mathrm{CTA}}=\mathrm{CTA}_{0} / M_{2_{0}}$. In Fig. 2 is presented the time evolution of weight average molecular weight in a batch reactor with $y_{M_{2}}=0.05$. 


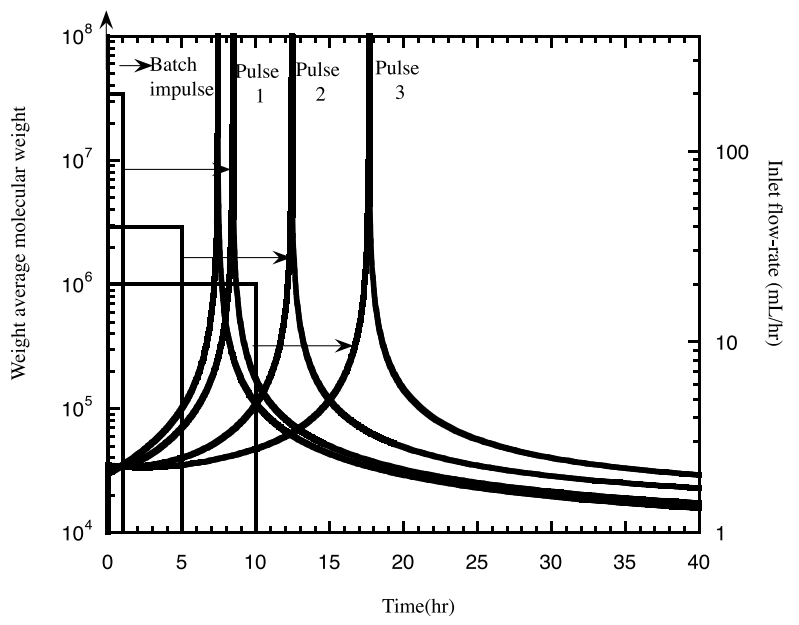

Fig. 2. Pulse feed of the divinyl monomer in a semibatch reactor and its influence on the time evolution of the weight average molecular weight.

\section{Semibatch operation}

\subsection{Feed policies for the divinyl monomer}

In this section, it is analyzed the dynamics of the average molecular weights and weight fraction of sol in a semibatch reactor with all of the monovinyl monomer initially charged in the reactor and considering different ways of feeding the divinyl monomer. This is an obvious choice for operating semibatch reactors in cross-linking polymerizations when it is desired to delay gelation without the presence of CTA.

For the purpose of comparison with batch reactors, it is defined the variable equivalent batch composition time of feed which represents the time of addition of the divinyl monomer necessary to have in the reactor the same number of moles of each monomer relatively to the correspondent batch operation. The most obvious way of attaining this goal is by using a pulse feed of the divinyl monomer as described by Eq. (21):

$Q_{\mathrm{F}}(t)=Q_{0}\left[H(t)-H\left(t-t_{\text {feed }}\right)\right]$

where $Q_{0}$ is a constant inlet flow rate, $t_{\text {feed }}$ is the time of feed and $H(t)$ is the Heaviside unit step function. For a fixed inlet concentration of the divinyl monomer and a reference number of moles of divinyl monomer that must be fed to the reactor, the time of feed can be calculated for a desired value of the inlet flow rate (and vice-versa). It can be also assured that the final reaction volume is the same of the correspondent batch operation. For instance, starting the semibatch operation with a reaction volume of $V_{0}=0.8 \mathrm{dm}^{3}$ and concentrations of monovinyl monomer and initiator $M_{1_{0}}=5 \mathrm{~mol} \mathrm{dm}^{-3}, I_{0}=$ $0.1 \mathrm{~mol} \mathrm{dm}^{-3}$ and feeding the reactor with divinyl monomer at concentration of $M_{2 \mathrm{~F}}=1 \mathrm{~mol} \mathrm{dm}^{-3}$ during $t_{\text {feed }}=1 \mathrm{~h}$ with an inlet flow rate of $Q_{0}=200 \mathrm{ml} / \mathrm{h}$ it is obtained the correspondence with a batch operation starting with $V_{0}=1 \mathrm{dm}^{3}, M_{1_{0}}=4 \mathrm{~mol} \mathrm{dm}^{-3}, M_{2_{0}}=0.2 \mathrm{~mol} \mathrm{dm}^{-3}$ and $I_{0}=0.08 \mathrm{~mol} \mathrm{dm}^{-3}$. Different pulse feed policies can be generated by varying the time of feed and consequently the inlet flow rate in order to have the same reference batch operation. These principles were used in the simulations presented in Fig. 2. Four different pulse feeds were considered with times of addition $t_{\text {feed }}=1 \mathrm{~s}$, and 1, 5 and $10 \mathrm{~h}$ with correspondent inlet flow rates of $Q_{0}=7.2 \times 10^{5}$, 200,40 and $20 \mathrm{ml} / \mathrm{h}$. The first situation was chosen in order to simulate an impulse feed to the reactor and, as expected, it reproduces the batch operation (Fig. 2). In the other cases, it can be observed that a slow feed of the divinyl monomer has a strong effect in delaying gelation and in the dynamics of time change of average molecular weights. So, the operation in semibatch reactors is an alternative to the use of a CTA in order to prevent gelation or to the control the properties of networks produced.

The comparison with batch operation was also performed considering a ramp feed policy of the divinyl monomer, namely an ascending ramp, as described by Eq. (22):

$Q_{\mathrm{F}}(t)=\alpha t\left[H(t)-H\left(t-t_{\text {feed }}\right)\right]$

Or a descending ramp (Eq. (23)):

$Q_{\mathrm{F}}(t)=\alpha\left(t_{\text {feed }}-t\right)\left[H(t)-H\left(t-t_{\text {feed }}\right)\right]$

The parameter $\alpha$ is calculated in order to attain a batch composition equivalence when the ramp feed is stopped at $t=t_{\text {feed. }}$. For the reference batch system with $y_{M_{2}}=0.05$ and a divinyl monomer feed concentration of $M_{\mathrm{F}_{\mathrm{F}}}=1 \mathrm{~mol} \mathrm{dm}^{-3}$, it must be $\alpha=0.016 \mathrm{dm}^{3} \mathrm{~h}^{-2}$ when $t_{\text {feed }}=5 \mathrm{~h}$. Once again, the semibatch operation must start with the reactor charged with monovinyl monomer and initiator with concentrations $M_{1_{0}}=5$ and $I_{0}=0.1 \mathrm{~mol} \mathrm{dm}^{-3}$ and a reaction volume of $V_{0}=0.8 \mathrm{dm}^{3}$. The influence of this kind of monomer feed policies on the dynamics of change of the weight average molecular weight and weight fraction of sol is presented in Figs. 3 and 4.

A cyclic feed of the divinyl monomer was also simulated by considering a sinusoidal variation of the inlet flow rate described by Eq. (24):

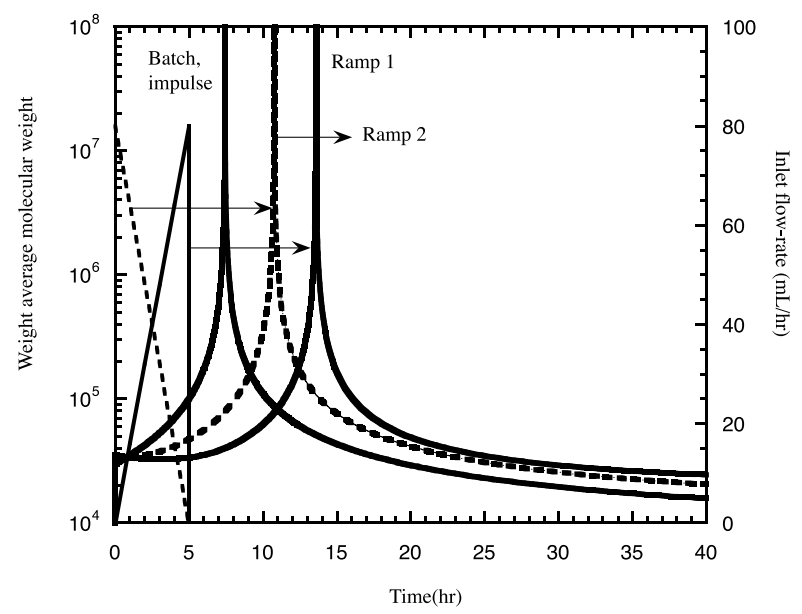

Fig. 3. Ramp feed of the divinyl monomer in a semibatch reactor and its influence on the time evolution of weight average molecular weight. 


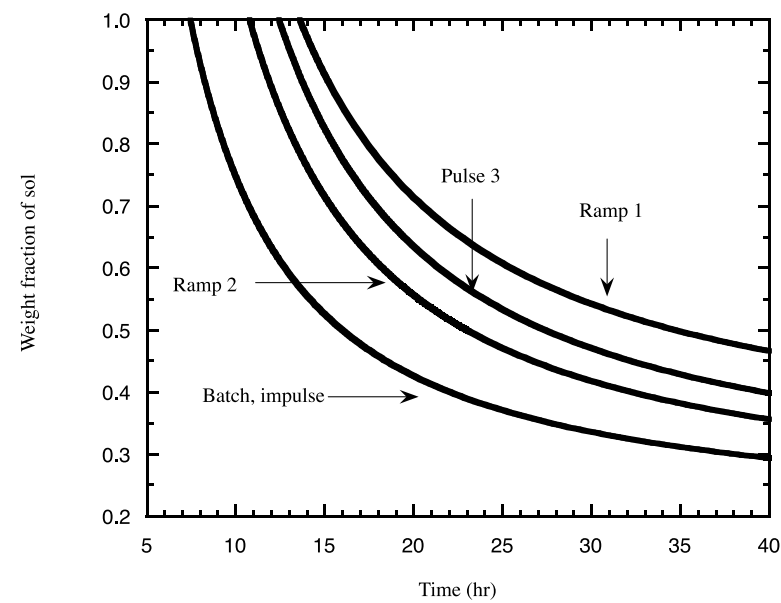

Fig. 4. The influence of different feed policies of the divinyl monomer on the time evolution of weight fraction of sol.

$Q_{\mathrm{F}}(t)=\beta|\sin (\omega t)|\left[H(t)-H\left(t-t_{\text {feed }}\right)\right]$

For a frequency $\omega=\pi \mathrm{rad} \mathrm{h}^{-1}$ and a feed time $t_{\text {feed }}=5 \mathrm{~h}$, following the principles before presented, it must be $\beta=$ $0.02 \pi \mathrm{dm}^{3} \mathrm{~h}^{-1}$.

A second kind of sinusoidal variation was simulated by considering the feed rate given by Eq. (25):

$Q_{\mathrm{F}}(t)=\beta[1-\sin (\omega t)]\left[H(t)-H\left(t-t_{\text {feed }}\right)\right]$

With $\omega=\pi \mathrm{rad} \mathrm{h}^{-1}$ and $t_{\text {feed }}=3 \mathrm{~h}$ one must set a value of $\beta=((0.2 \pi) /(3 \pi-2)) \mathrm{dm}^{3} \mathrm{~h}^{-1}$. In Fig. 5 are presented the simulation results for these cyclic feed policies and it is clearly seen their influence on the time evolution of the average properties of the network. The analysis of this nonconventional feed policies is important to verify the ability of the simulation method to deal with it.

The number of possibilities for different feed strategies is obviously very large and a last case study where the divinyl monomer is also added after gelation was considered. In this case the inlet flow-rate follows a sequence of rectangular

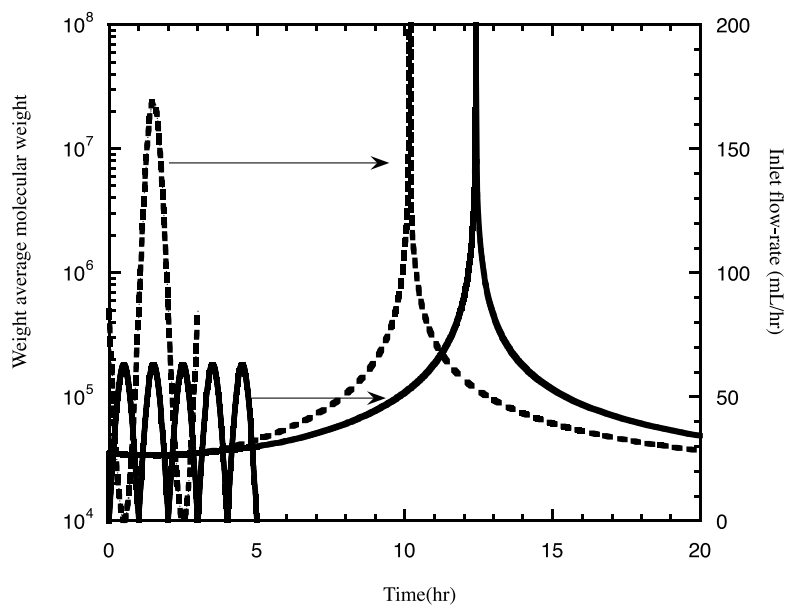

Fig. 5. The influence of different cyclic feed policies of the divinyl monomer on the time evolution of weight average molecular weight. waves, which is represented by Eq. (26):

$Q_{\mathrm{F}}(t)=Q_{0} \sum_{k=0}^{N-1}\left[H\left(t-t_{2 k}\right)-H\left(t-t_{2 k+1}\right)\right]$

where $N$ is the number of waves considered and $t_{2 k}=t_{\text {feed }}+$ $2 k T$ is the feed start time for the $k+1$ wave. In this case, $t_{\text {feed }}$ represents the feed start time of the first wave and $T$ is the period of feed for each wave. For instance, for reproducing the batch composition used in the other examples, with $N=$ 4 and $T=2 \mathrm{~h}, Q_{0}=25 \mathrm{ml} / \mathrm{h}$ must hold.

In Fig. 6 can be observed the strong influence of these kind of monomer feed policies in the dynamics of the change of weight average molecular weight.

\subsection{Feed policies for the monovinyl monomer}

In this case, it is supposed that all amounts of the divinyl monomer and initiator are initially charged in the reactor. Some amount of the monovinyl monomer is also present in the start of the polymerization and the remaining is gradually fed to the reactor. The simulations were also performed in order to keep the equivalence with batch composition. In this way, to follow the batch polymerization with $y_{M_{2}}=0.05$ and supposing that $25 \%$ of the monovinyl monomer is initially in the reactor, the polymerization must start with $M_{1_{0}}=2.5 \mathrm{~mol} \mathrm{dm}^{-3}, M_{2_{0}}=0.5 \mathrm{~mol} \mathrm{dm}^{-3}$ and $I_{0}=0.2 \mathrm{~mol} \mathrm{dm}^{-3}$ if the feed concentration of the monovinyl monomer is $M_{1_{\mathrm{F}}}=5 \mathrm{~mol} \mathrm{dm}^{-3}$. Starting the semibatch operation with a reaction volume of $V_{0}=0.4 \mathrm{dm}^{3}$ and feeding the reactor during $t_{\text {feed }}=10 \mathrm{~h}$ with an inlet flow rate of $Q_{0}=60 \mathrm{ml} / \mathrm{h}$ it is obtained the correspondence with a batch operation starting with $V_{0}=1 \mathrm{dm}^{3}, M_{1_{0}}=4 \mathrm{~mol} \mathrm{dm}{ }^{-3}$, $M_{2_{0}}=0.2 \mathrm{~mol} \mathrm{dm}^{-3}$ and $I_{0}=0.08 \mathrm{~mol} \mathrm{dm}^{-3}$. In this case, the semibatch operation is characterized by an initial molar ratio of the two monomers equal to $y_{M_{20}}=0.2$. Similar relations can be established, for instance, in the cases $y_{M_{20}}=0.1$

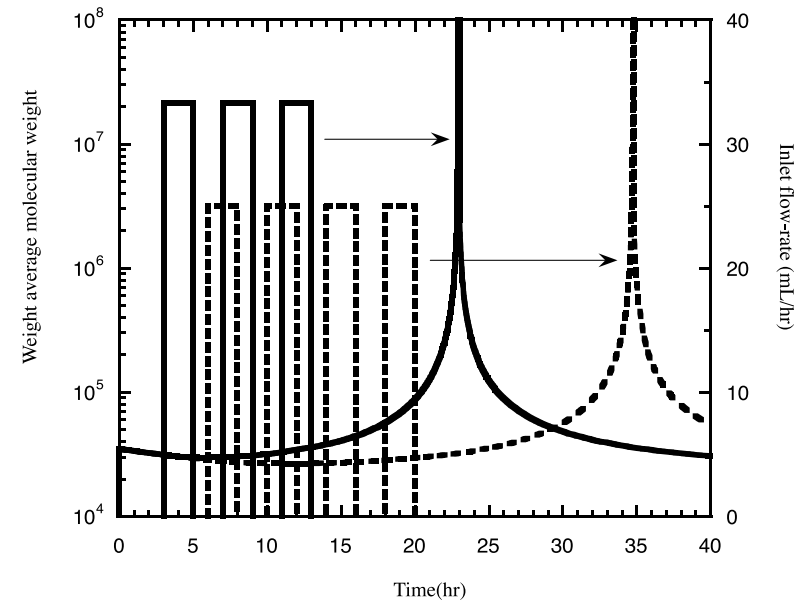

Fig. 6. The influence of different rectangular wave feed policies of the divinyl monomer on the time evolution of the weight average molecular weight. 
(50\% of the monovinyl monomer initially in the reactor) or $y_{M_{20}}=0.067$ (75\% of the monovinyl monomer initially in the reactor).

As discussed in Section 6.1, several monomer feed policies can be obtained by varying the function $Q_{\mathrm{F}}(t)$. In Fig. 7 it is represented the dynamics of the weight average molecular weight for a pulse feed of the monovinyl monomer with different initial compositions and supposing that the batch composition equivalence is attained always with a feed time of $10 \mathrm{~h}$. Only a small variation can be observed in the time of gelation of the three systems. The difference relatively to the batch operation is also very small. Nevertheless, this variation is not regular with the initial composition/monomer feed rate due to the kinetics of the polymerization, namely because the divinyl monomer is faster incorporated in the polymer by propagation with a free radical from the monovinyl monomer.

The influence of the reactivity ratios becomes very clear if the monovinyl monomer is not added from the beginning of the polymerization, as represented in Fig. 8 for a rectangular wave feed. It should be noticed that, in spite of the high fraction of the divinyl monomer in the initial composition $\left(y_{M_{20}}=0.2\right)$, gelation is delayed relatively to the pulse feed policy (and batch operation) due to kinetics of the polymerization. It is also interesting to notice that in this case some amount of the reagents is fed to the reactor after gelation.

The results just presented show that major differences in the dynamics of time change of average molecular weights relatively to the batch operation are observed (with this kinetic scheme) when the feed of the semibatch reactor is only the divinyl monomer.

\subsection{Feed policies for the initiator and chain transfer agent}

It can be qualitatively predicted that the addition of the chain transfer agent to the reactor in some feed policy

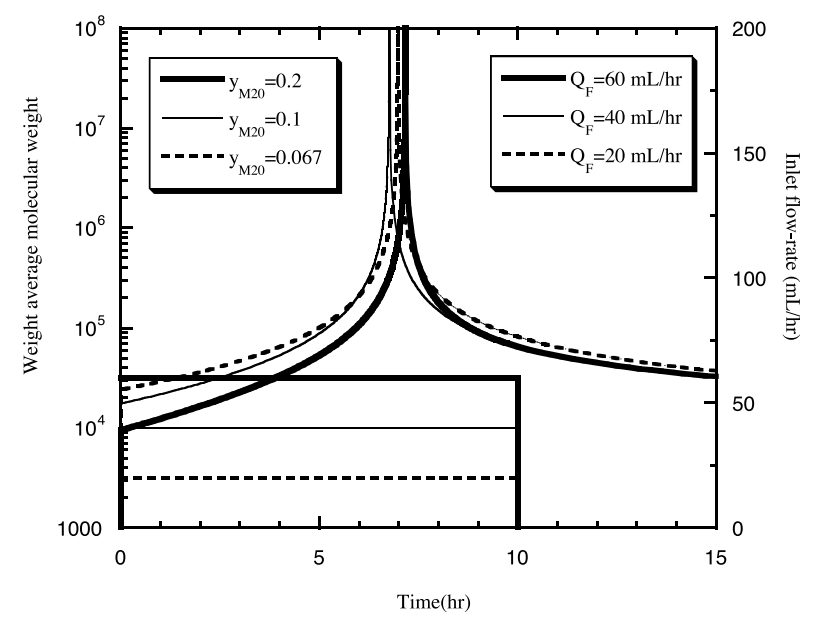

Fig. 7. Pulse feed of the monovinyl monomer in a semibatch reactor and its influence on the time evolution of the weight average molecular weight for different initial compositions.

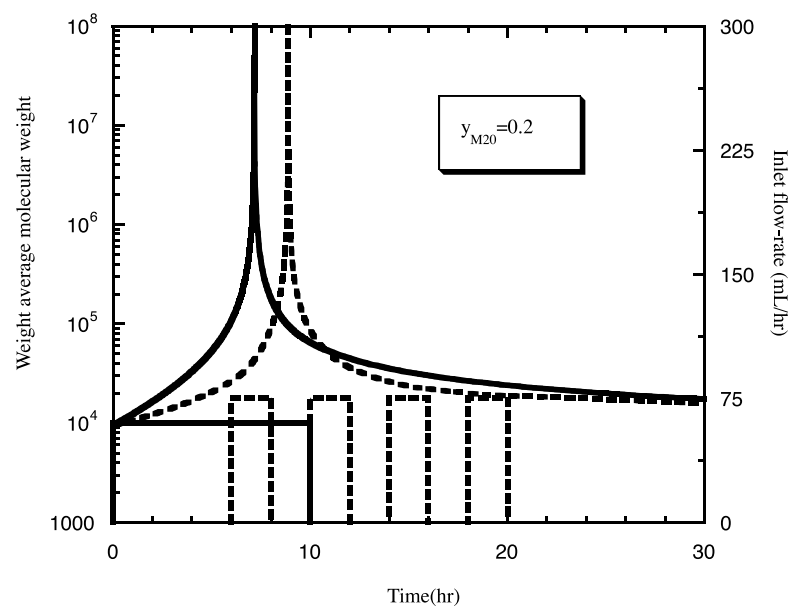

Fig. 8. Pulse and rectangular wave feed of the monovinyl monomer in a semibatch reactor and its influence on the time evolution of the weight average molecular weight for an initial composition described by $y_{M_{20}}=0.2$.

different from the batch operation will affect the dynamics of the average properties of the polymer. Namely, it is expected to get an earlier gelation comparatively to the batch operation with the same amount of CTA. To quantify these aspects, it was simulated the semibatch operation starting with the whole amounts of the both monomers in the reactor and feeding the CTA with variable strategies. Again, in the end of feed an equivalent batch composition can be attained. For instance, to have an equivalence with the batch operation with $y_{M_{2}}=0.05$ and $y_{\mathrm{CTA}}=0.1$, feeding the reactor with a CTA solution with concentration $\mathrm{CTA}_{\mathrm{F}}=$ $0.1 \mathrm{~mol} \mathrm{dm}^{-3}$ with a pulse input during $4 \mathrm{~h}$, the rate of feed must be $Q_{0}=50 \mathrm{ml} / \mathrm{h}$. With a starting reaction volume of $V_{0}=0.8 \mathrm{dm}^{3}$ and concentrations $M_{1_{0}}=5 \mathrm{~mol} \mathrm{dm}^{-3}, M_{2_{0}}=$ $0.25 \mathrm{~mol} \mathrm{dm}^{-3}$ and $I_{0}=0.1 \mathrm{~mol} \mathrm{dm}^{-3}$ an equivalence with the batch composition is obtained in the end of feed.

In a different strategy, the CTA can be continuously fed to the reactor in a step policy after some time of polymerization. In this case the batch composition equivalence is not attained. In Fig. 9 can be found the quantification of the influence of different feed policies of the CTA in the dynamics of the average molecular weight of the polymer. The calculations with the pulse feed were obtained according to the principles before presented and the step feed was simulated with a feed rate $Q_{0}=75 \mathrm{ml} / \mathrm{h}$ and an inlet concentration of CTA, CTA $=0.1 \mathrm{~mol} \mathrm{dm}^{-3}$. In these conditions, and as expected, with the step feed a delay in gelation is observed and with a pulse feed an earlier gelation occurs, relatively to the correspondent batch operation.

Similar simulations were performed in order to analyze de influence of the initiator feed policies in the dynamics of the properties of the resulting polymer. The most interesting situation is the feed of the initiator by a sequence of rectangular waves, which represents something similar to PLP but applied to non-linear copolymerizations. In Fig. 10 


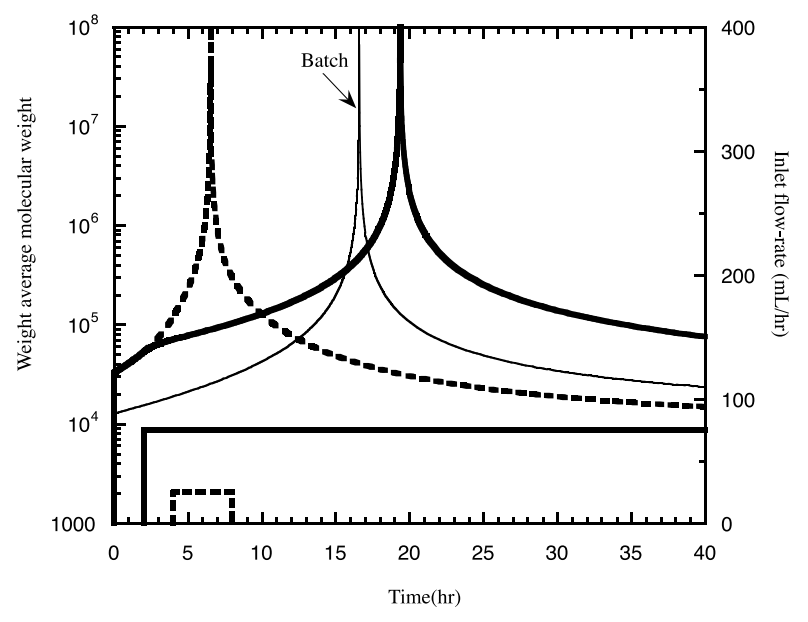

Fig. 9. Pulse and step feed of the chain transfer agent in a semibatch reactor and its influence on the time evolution of the weight average molecular weight for an initial composition described by $y_{M_{2}}=0.05$.

are reproduced the results of this case study for $N=5$ and $T=1 \mathrm{~h}$ when the semibatch polymerization starts with both monomers initially charged to the reactor. The concentration of the initiator in the inlet stream was fixed at $I_{\mathrm{F}}=$ $0.4 \mathrm{~mol} \mathrm{dm}^{-3}$ and a final batch composition equivalence is attained relatively to the system with $y_{M_{2}}=0.05$ by using an inlet flow rate of $Q_{0}=40 \mathrm{ml} / \mathrm{h}$. In this case, an intermediate decrease in weight average molecular weight is predicted due to the addition of the initiator. Nevertheless, an earlier gelation should occur relatively to the correspondent batch operation.

The influence of primary cyclization was also put into evidence in semibatch operation. In Fig. 11 is presented the weight average molecular weight for a pulse feed of the divinyl monomer in a semibatch reactor for different values of $k_{\text {cyc }}$. These calculations are based upon the same principles already presented for semibatch operation in the absence of cyclization. As expected, as $k_{\text {cyc }}$ becomes larger,

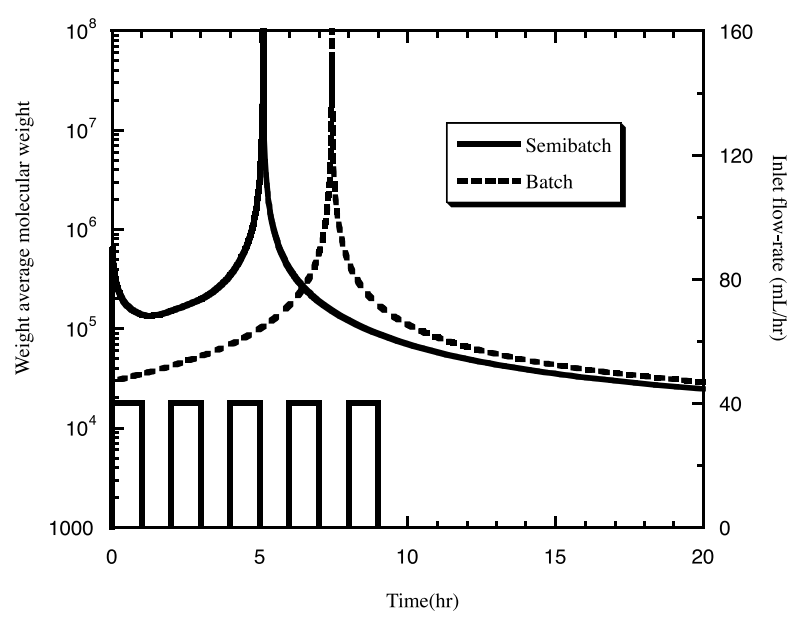

Fig. 10. Rectangular wave feed of the initiator in a semibatch reactor and its influence on the time evolution of the weight average molecular weight for an initial composition described by $y_{M_{2}}=0.05$.

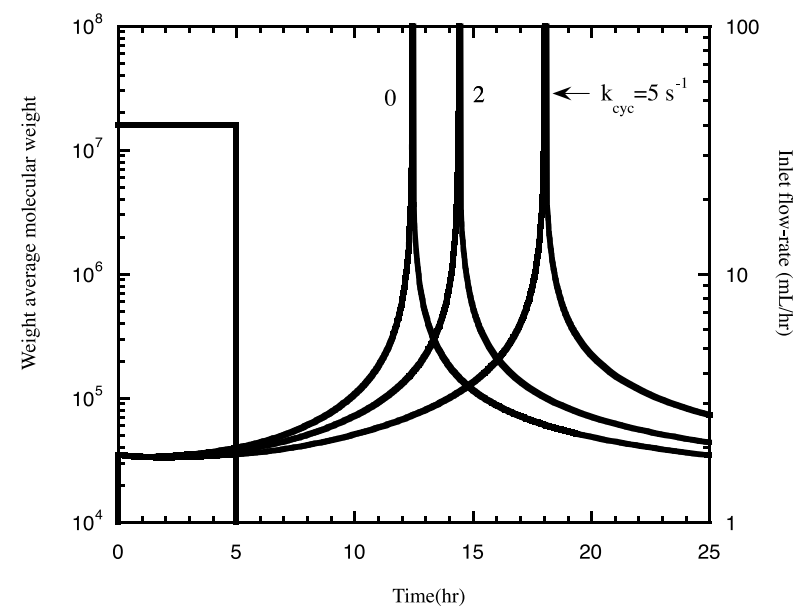

Fig. 11. The influence of primary cyclization on the weight average molecular weight for a pulse feed of the divinyl monomer in a semibatch reactor.

there is a further delay in gelation and the network properties are also modified. Analogous simulations have been carried out for a pulse feed of the monovinyl monomer in a semibatch reactor (Fig. 12). Comparing Fig. 7 with Fig. 12, it is concluded that primary cyclization strongly affects the polymer properties also when the feed policy consists in the addition of the monovinyl monomer. This can be explained through the increase of cyclization when the relative amount of divinyl monomer increases in the reaction mixture.

\section{Conclusions}

In this work, it was presented a theoretical study about free radical crosslinking copolymerizations in semibatch reactors with the inclusion of primary cyclization. The major distinctive features of this analysis are:

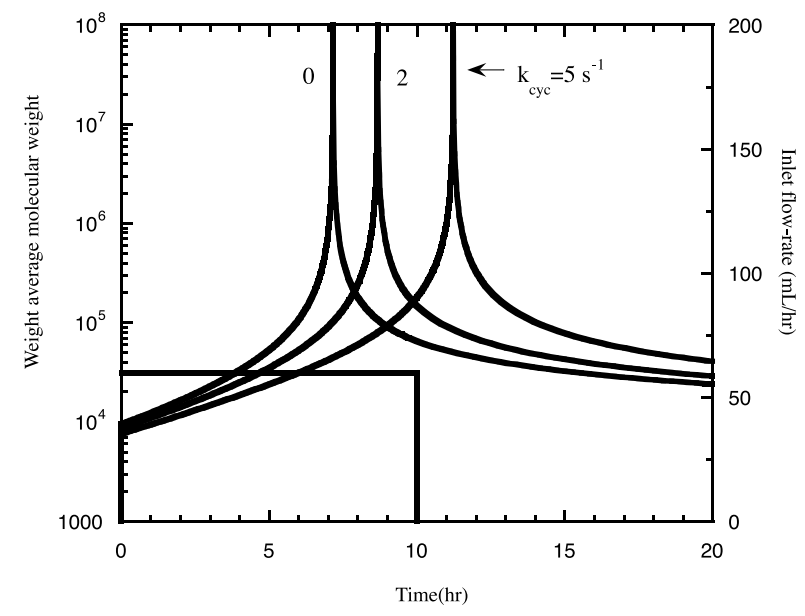

Fig. 12. The influence of primary cyclization on the weight average molecular weight for a pulse feed of the monovinyl monomer in a semibatch reactor. 
- The application of a simulation method that avoids a set of approximating conditions with widespread use in modeling of non linear free radical polymerizations;

- The extension of the analysis of crosslinking copolymerizations in semibatch reactors past gel point;

- The consideration of a detailed kinetic scheme with distinction of five macroradicals and four monomers (two real monomers plus two kinds of pendant double bonds). If cyclization is absent a parallelism with a terpolymerization can be established;

- The possibility for accounting primary cyclization in a purely kinetic fashion.

The dynamics of time change of weight fraction of sol and average molecular weights in semibatch reactors have been presented for a different set of monomer feed policies. The use of non-conventional feed strategies testifies the ability of the simulating method to deal with non-classic simulation problems.

The results obtained with this kinetic scheme have shown that different divinyl monomer feed policies have a strong effect in the properties of the resulting polymers. On the contrary, only small differences relatively to batch operation are predicted when the monovinyl monomer is fed to the reactor if cyclization is absent. The time feed of chain transfer agent or initiator can also be used to have a better control on the network structure. These findings can be used to implement new design ideas in polymer networks production.

The improved mathematical exactness of the present method and the possibility to consider detailed complex schemes should also be useful for obtaining better estimates of the kinetic parameters involved in this kind of polymerizations.

\section{References}

[1] Hild G, Okasha R. Makromol Chem 1985;186:93-110.

[2] Hild G, Okasha R. Makromol Chem 1985;186:389-406.

[3] Hild G, Okasha R, Rempp P. Makromol Chem 1985;186:407-22.

[4] Li WH, Hamielec AE, Crowe CM. Polymer 1989;30:1513-7.

[5] Naghash HJ, Okay O, Yildirim H. J Appl Polym Sci 1995;56:477-83.

[6] Nyhus AK, Hagen S, Berge A. J Polym Sci (A) 1999;37:3345-59.

[7] Zetterlund PB, Yamazoe H, Yamada B. Polymer 2002;43:7027-35.

[8] Elliott JE, Nie J, Bowman CN. Polymer 2003;44:327-32.

[9] Flory PJ. Principles of polymer chemistry. Ithaca, New York: Cornell University Press; 1953 [chapter 9].
[10] Bamford CH, Tompa H. Trans Faraday Soc 1954;50:1097-115.

[11] Tobita H, Hamielec AE. Makromol Chem Macromol Symp 1988; 20/21:501-43.

[12] Tobita H, Hamielec AE. Macromolecules 1989;22:3098-105.

[13] Hutchinson RA. Polym React Eng 1993;1:521-77.

[14] Arriola DJ. Modeling of addition polymerization systems. PhD Thesis, USA: Univ. of Wisconsin; 1989. p. 42.

[15] Pladis P, Kiparissides C. Chem Eng Sci 1998;53:3315-33.

[16] Baltsas A, Achilias DS, Kiparissides C. Macromol Theory Simul 1996;5:477-97.

[17] Kurdikar DL, Somvarsky J, Dušek K, Peppas NA. Macromolecules 1995;28:5910-20

[18] He JP, Zhang HD, Chen JM, Yang YL. Macromolecules 1997;30: 8010-8.

[19] Tobita H. Macromolecules 1993;26:836-41.

[20] Tobita H. Macromolecules 1993;26:5427-35.

[21] Bowman CN, Peppas NA. Chem Eng Sci 1992;47:1411-9.

[22] Elliott JE, Bowman CN. Macromolecules 1999;32:8621-8.

[23] Prescott J. Macromolecules 2003;36:9608-21.

[24] Wen M, Scriven LE, McCormick A. Macromolecules 2003;36: 4140-50.

[25] Tobita H, Hamielec AE. Polymer 1992;33:3647-57.

[26] Tobita H, Tani N, Dakai T. J Polym Sci, Part B: Polym Phys 2000;38: 2342-50.

[27] Teymour F, Campbell JD. Macromolecules 1994;27:2460-9.

[28] Papavasiliou G, Teymour F. Macromol Theory Simul 2003;12:543-8.

[29] Iedema PD, Hoefsloot HCJ. Macromol Theory Simul 2002;11: 410-28.

[30] Iedema PD, Hoefsloot HCJ. Macromolecules 2004;37:10155-64.

[31] Costa MRPFN, Dias RCS. Chem Eng Sci 1994;49:491-516.

[32] Costa MRPFN, Dias RCS. Macromol Theory Simul 2003;12:560-72.

[33] Dias RCS, Costa MRPFN. Macromolecules 2003;36:8853-63.

[34] Costa MRPFN, Dias RCS. Chem Eng Sci 2005;60:423-46.

[35] Dias RCS, Costa MRPFN. Macromol Theory Simul 2005;14:243-55.

[36] Matsumoto A. Prog Polym Sci 2001;26:189-257.

[37] Litvinenko GI, Kaminsky VA. Prog React Kinet 1994;19:139-93.

[38] de Kock JBL, Van Herk AM, German AL. J Macromol Sci, Polym Revs 2001;C41:199-252.

[39] Keramopoulos A, Kiparissides C. Macromolecules 2002;35:4155-66.

[40] Berchtold KA, Lovestead TM, Bowman CN. Macromolecules 2002; 35:7968-75.

[41] Andrzejewska E. Prog Polym Sci 2001;26:605-65.

[42] Matsumoto A. Adv Polym Sci 1995;123:41-80.

[43] Matsumoto A, Ueda A, Aota A, Ikeda JI. Eur Polym J 2002;38: 1777-82.

[44] Bouvier-Fontes L, Pirri R, Asua JM, Leiza JR. Macromolecules 2005; 38:1164-71.

[45] Landin DT, Macosko CW. Macromolecules 1988;21:846-51.

[46] Okay O, Naghash HJ. Macromol Theory Simul 1995;4:967-81.

[47] Okay O, Kurz M, Lutz K, Funke W. Macromolecules 1995;28: 2728-37.

[48] Okay O. J Appl Polym Sci 1999;74:2181-95.

[49] Elliott JE, Anseth JW, Bowman CN. Chem Eng Sci 2001;56:3173-84. 